\title{
Reference values for blood pressure of healthy Sri Lankan Tamil children in the Jaffna district
}

\author{
*M Sooriyakanthan ${ }^{1}$, N Vasikaran ${ }^{2}$, A Puvana ${ }^{2}$, K Shobijah $^{2}$, MPF Nusra $^{2}$, K Sivapalan $^{3}$
}

Sri Lanka Journal of Child Health, 2018; 48: 257-264

\begin{abstract}
Introduction: Increasing trend of childhood obesity leads to high blood Pressure (BP) in children and adolescents. Ethnic differences in BP have been reported. Normal BP values of Sri Lankan Tamil children are not available.
\end{abstract}

Objective: To measure BP of healthy Sri Lankan Tamil children in the Jaffna district to get normal BP values and to correlate them with anthropometric measurements.

Method: A population based descriptive cross sectional study was carried out among children and adolescents (950 boys, 972 girls) aged 6 to 18 years in schools in Jaffna district. Cluster sampling was applied to classrooms in the schools. The classes were selected by systematic random sampling. Age, height, weight, waist circumference and hip circumference were taken. Body mass index, waist hip ratio and waist height ratio were calculated. BP was measured with a mercury sphygmomanometer. Pubertal stage was assessed with a self-administered Tanner staging scale.

Results: Mean of the systolic blood pressure (SBP) and diastolic blood pressure (DBP) of boys and girls increased from 98/70 $\mathrm{mm} \mathrm{Hg}$ and 99/70 $\mathrm{mm} \mathrm{Hg}$ to $107 / 73 \mathrm{~mm} \mathrm{Hg}$ and $107 / 73 \mathrm{~mm} \mathrm{Hg}$ until 10 years, decreased slightly up to 13 years $(101 / 64 \mathrm{~mm} \mathrm{Hg}$ and $102 / 63 \mathrm{~mm} \mathrm{Hg})$ and increased until 18 years to $119 / 76 \mathrm{~mm} \mathrm{Hg}$ and $111 / 70 \mathrm{~mm} \mathrm{Hg}$. From the age of 15 years, boys had higher SBP and DBP than girls $(\mathrm{p}<0.05)$. SBP had significant $(p<0.001)$ positive correlations with pubertal staging and all measured anthropometric parameters. Highest correlation was observed with weight (0.522). A normogram for BP was constructed with age and height.

${ }^{1}$ Lecturer, ${ }^{2}$ Demonstrator, ${ }^{3}$ Associate Professor, Department of Physiology, Faculty of Medicine, University of Jaffna, Sri Lanka

*Correspondence: mathu1481@yahoo.com

(Received on 11 December 2017: Accepted after revision on 19 January 2018)

The authors declare that there are no conflicts of interest

Funding: University Research Grant, 2015

Open Access Article published under the Creative

Commons Attribution CC-BY (CC) (P) License
Statistically significant $(\mathrm{p}<0.05)$ increases in SBP and DBP were observed from pubertal stage 3 to pubertal stage 4 in both boys and girls. Jaffna boys up to 15 years and girls up to 10 years have higher BP than Sinhalese children at a purana village. After these ages BP values were lower than in Sinhalese children.

Conclusion: BP of healthy Sri Lankan Tamil children in the Jaffna district has significant correlations with anthropometric measurements and Tanner staging.

DOI: http://dx.doi.org/10.4038/sljch.v47i3.8548

(Key words: Blood pressure, Sri Lankan Tamil children)

\section{Introduction}

High blood pressure (BP) is a powerful, constant, and independent risk factor for cardiovascular and renal diseases ${ }^{1}$. The definition of hypertension is based on normative distribution of $\mathrm{BP}^{2}$. Increasing trend of childhood obesity leads to high BP in children and adolescents ${ }^{3}$. Nowadays behavioural patterns of communities are changing with urbanization and industrialization leading to sedentary life styles and consumption of high fat and energy dense diets which cause obesity. Hypertension is the common childhood condition seen in obese and overweight individuals. Hereditary and environmental factors also influence body mass index (BMI) and body fat which may influence the $\mathrm{BP}^{4}$. The biological rationale for relating measures of central adiposity to cardiovascular disease (CVD) risk is that the abdominal adipose tissue is positively associated with waist circumference (WC) and waist-hip ratio (WHR). As the WHR increases, BP increases ${ }^{5}$. Further, BP is important in childhood conditions such as acute glomerular nephritis and dehydration.

Unlike the practice of measuring BP in all adult patients and advising to monitor it regularly, BP in children is measured only when there is a suspicion of alteration in BP. In such instances, interpretation of the measured BP can be of value only if reference norms for the age, sex, height and ethnicity are available. Therefore, normal values of the Jaffna population should be known to be able to diagnose hypertension among children and adolescents in this region. The only report available 
on BP values of Sri Lankan children is from a study on inhabitants of a "purana" village of Sri Lanka in $1991^{6}$. Data on the BP of healthy Sri Lankan Tamil children are not available. Mean BP values were higher in Afro-Caribbean and South Asian men than their white counterparts ${ }^{7}$. Among South Asians, Indians have a slightly higher BP, Pakistanis have a slightly lower BP and Bangladeshis have a much lower $\mathrm{BP}^{8}$. This shows that ethnic differences in BP exist.

\section{Objectives}

This study aimed at measuring BP in healthy Tamil children in Jaffna to get normal BP values and to correlate them with anthropometric measurements.

\section{Method}

This was a population based descriptive cross sectional study. Children aged 6 to 18 years were recruited from schools in Jaffna district as education is compulsory in Sri Lanka. Cluster sampling was applied to classrooms in schools of Jaffna District. Each cluster was a classroom consisting of around 30 students. Number of clusters in each grade was selected according to the percentage of total students in that grade. Classes were selected by systematic random sampling.

Ethical clearance for the study was obtained from the Ethical Review Committee of the Faculty of Medicine, University of Jaffna. Permission was obtained from the Provincial Director of Education, each Zonal Director of Education, School Principals and Class Teachers prior to the study. Informed written consent was obtained from all parents and assent from adolescents 14 years or older. The information sheet and consent form were given to the participants on the day before data collection to get the consent from the parents.

A self-administered questionnaire, which included general information and disease history of the participant, was issued to one parent of each participant. Children with a history of diabetes and children diagnosed to have any type of CVD were excluded. Anyone who had signs and symptoms suggestive of anaemia, cardiac or renal disorders on medical examination during data collection and children having the habit of smoking and alcohol intake were also excluded.

Age was calculated in years as on the last birthday. Body height and weight were measured according to a standardized protocol to the nearest $0.1 \mathrm{~cm}$ and $0.1 \mathrm{~kg}$ respectively by using a portable device (Seca). BMI was calculated from measured body weight and height. WC was measured by placing the non-elastic measuring tape midway between the uppermost border of the iliac crest and lower border of costal margin while subject was standing.
Measurement was taken at the end of expiration. Hip circumference (HC) was measured by placing a non-elastic tape over the widest part of the hip 9 .

The procedure was explained thoroughly to the participants before recording blood pressure and sufficient time was given to allay anxiety and fear. Participants were in a sitting position with the legs uncrossed. BP was measured in right arm by the auscultatory method using a standard mercury sphygmomanometer. The cuff bladder was wide enough to cover at least $80 \%$ of the arm and long enough to encircle the arm. The cuff was inflated to at least $30 \mathrm{~mm} \mathrm{Hg}$ above the point at which radial pulse disappeared. The cuff was deflated at a rate of $2-3 \mathrm{~mm} \mathrm{Hg} /$ second. The first Korotkoff sound was taken as indicative of systolic pressure and the disappearance of the sound was the diastolic blood pressure. Three readings were taken at 5 minute intervals. As the first reading was usually higher, the average of second and third readings was considered for analysis.

Participants aged above 8 years were given the self-administered questionnaire with the Tanner staging scale which consisted of pictures of genital organs and breasts at different stages. Participants had to compare their genital organs and breasts with that on the Tanner staging scale and identify and mark the pubertal stage in the questionnaire. This was done in a confidential place separately for males and females after giving clear instructions. One investigator of the same sex was available to clear doubts regarding marking the pubertal stage.

Data analysis was done by Statistical Package for Social Science. Independent t-test was used to compare significance of difference between means. Pearson's correlation coefficient (r) was used to evaluate relationship between $\mathrm{BP}$ and height, weight, BMI, WC, HC, WHR and Tanner staging.

\section{Results}

There were 950 boys and 972 girls. Anthropometric measurements of the study population are summarized in Table 1 .

Mean height of boys and girls of each age showed statistically significant $(p<0.05)$ differences beyond 13 years of age. Differences in the mean weight of boys and girls were statistically significant $(p<0.05)$ beyond 16 years of age.

Pearson correlation of height, weight, BMI, WC and $\mathrm{HC}$ with age were $0.931,0.817,0.558,0.620$ and 0.784 respectively in boys and $0.886,0.810$, $0.629,0.625$ and 0.814 respectively in girls. All these were statistically significant at $p<0.001$. Blood pressure values of boys and girls in each age group are summarised in Table 2. 
Table 1: Anthropometric measurements of the study population

\begin{tabular}{|c|c|c|c|c|c|c|c|}
\hline \multirow{2}{*}{$\begin{array}{c}\text { Age } \\
\text { (years) }\end{array}$} & \multirow[t]{2}{*}{$\mathrm{n}$} & \multicolumn{5}{|c|}{ Mean (SD) } & \multirow{2}{*}{$\begin{array}{c}\text { Waist/Hip } \\
\text { ratio }\end{array}$} \\
\hline & & Ht. (cm) & Wt. (kg) & BMI $\left(\mathrm{kg} / \mathrm{m}^{2}\right)$ & WC (cm) & HC (cm) & \\
\hline \multicolumn{8}{|l|}{ BOYS } \\
\hline 6 & 61 & $119.3(4.9)$ & $18.2(2.7)$ & $12.8(1.2)$ & $46.5(4.6)$ & $55.3(3.9)$ & $0.84(0.05)$ \\
\hline 7 & 79 & $123.7(5.6)$ & $20.6(4.5)$ & $13.3(2.1)$ & $48.6(6.0)$ & $57.7(6.1)$ & $0.84(0.05)$ \\
\hline 8 & 75 & $129.5(3.9)$ & $23.6(5.2)$ & $14.2(2.7)$ & $50.5(5.9)$ & $60.8(5.7)$ & $0.83(0.05)$ \\
\hline 9 & 81 & $132.1(4.8)$ & $25.3(6.0)$ & $14.4(2.5)$ & $52.2(7.0)$ & $62.3(6.6)$ & $0.84(0.05)$ \\
\hline 10 & 71 & $135.1(5.0)$ & $27.0(6.9)$ & $14.7(2.9)$ & $52.7(8.1)$ & $63.8(7.2)$ & $0.82(0.05)$ \\
\hline 11 & 76 & $141.4(7.3)$ & $32.6(9.4)$ & $16.1(3.9)$ & $58.9(11.8)$ & $68.7(9.3)$ & $0.85(0.06)$ \\
\hline 12 & 76 & $145.3(7.7)$ & $34.9(9.6)$ & $16.2(3.3)$ & $59.5(9.9)$ & $70.4(8.4)$ & $0.84(0.07)$ \\
\hline 13 & 76 & $151.4(8.2)$ & $38.5(8.1)$ & $16.7(2.7)$ & $62.2(8.5)$ & $73.8(7.3)$ & $0.84(0.06)$ \\
\hline 14 & 72 & $157.6(8.6)$ & $43.5(10.8)$ & $17.2(2.8)$ & $63.7(9.5)$ & $76.7(9.0)$ & $0.83(0.06)$ \\
\hline 15 & 92 & $163.6(6.8)$ & $47.6(10.0)$ & $17.7(2.9)$ & $64.6(8.3)$ & $79.0(7.6)$ & $0.81(0.06)$ \\
\hline 16 & 84 & $166.5(6.9)$ & $50.6(11.3)$ & $18.1(3.6)$ & $65.8(9.8)$ & 80.8 (7.9) & $0.81(0.06)$ \\
\hline 17 & 56 & $169.0(6.9)$ & $55.1(11.7)$ & $19.2(3.4)$ & $66.1(10.2)$ & $84.2(7.9)$ & $0.78(0.07)$ \\
\hline 18 & 51 & $171.4(6.1)$ & $56.9(8.8)$ & $19.4(3.1)$ & $67.4(7.8)$ & $84.7(6.8)$ & $0.80(0.06)$ \\
\hline \multicolumn{8}{|l|}{ GIRLS } \\
\hline 6 & 63 & $120.1(4.8)$ & $18.7(3.2)$ & $12.9(1.6)$ & $49.2(7.4)$ & $57.1(4.2)$ & $0.84(0.06)$ \\
\hline 7 & 75 & $123.9(4.6)$ & $20.4(3.8)$ & $13.2(1.9)$ & $49.8(5.1)$ & $59.1(4.9)$ & $0.84(0.05)$ \\
\hline 8 & 79 & $128.1(4.6)$ & $22.9(5.8)$ & $13.9(2.7)$ & $52.3(6.1)$ & $61.7(6.3)$ & $0.85(0.04)$ \\
\hline 9 & 79 & $132.5(4.9)$ & $26.0(6.0)$ & $14.7(2.5)$ & $53.7(7.0)$ & $63.9(6.4)$ & $0.84(0.05)$ \\
\hline 10 & 77 & $136.4(6.6)$ & $28.0(5.5)$ & $15.0(1.9)$ & $54.8(5.6)$ & 66.5 (5.6) & $0.82(0.04)$ \\
\hline 11 & 83 & $141.4(7.1)$ & $32.2(7.0)$ & $16.0(2.5)$ & $57.5(6.4)$ & $69.6(6.9)$ & $0.83(0.04)$ \\
\hline 12 & 72 & $148.2(7.5)$ & $37.7(10.3)$ & $17.0(3.7)$ & $60.1(9.1)$ & 74.4 (8.6) & $0.81(0.05)$ \\
\hline 13 & 78 & $151.2(7.2)$ & $38.9(7.7)$ & $17.0(2.5)$ & $60.2(6.3)$ & $75.1(6.5)$ & $0.80(0.05)$ \\
\hline 14 & 84 & $154.2(6.3)$ & $43.5(9.4)$ & $18.1(3.4)$ & $62.8(7.7)$ & $79.5(7.6)$ & $0.79(0.04)$ \\
\hline 15 & 99 & $155.9(6.4)$ & $45.1(8.1)$ & $18.6(3.1)$ & $64.0(7.7)$ & $82.1(7.2)$ & $0.78(0.05)$ \\
\hline 16 & 77 & $157.9(5.7)$ & $48.6(7.9)$ & $19.6(2.7)$ & $65.1(6.3)$ & $84.4(6.5)$ & $0.77(0.04)$ \\
\hline 17 & 60 & $156.9(6.1)$ & $46.9(8.3)$ & $19.0(2.8)$ & $65.9(8.8)$ & $83.3(6.5)$ & $0.79(0.06)$ \\
\hline 18 & 46 & $158.8(5.3)$ & $50.0(8.6)$ & $19.8(3.2)$ & $66.3(7.5)$ & 86.0 (7.4) & $0.77(0.05)$ \\
\hline
\end{tabular}

Ht. - height, Wt. - weight, BMI - body mass index, WC - waist circumference, HC - hip circumference, SD standard deviation, $n$ - number

Table 2: Blood pressure values of boys and girls in each age group

\begin{tabular}{|c|c|c|c|c|c|c|c|c|}
\hline \multirow{3}{*}{$\begin{array}{c}\text { Age } \\
\text { (years) }\end{array}$} & \multirow[t]{3}{*}{$\mathbf{n}$} & \multirow{2}{*}{\multicolumn{2}{|c|}{$\begin{array}{c}\text { BOYS } \\
\text { Mean (SD) }\end{array}$}} & \multirow[t]{3}{*}{ n } & \multirow{2}{*}{\multicolumn{2}{|c|}{$\begin{array}{c}\text { GIRLS } \\
\text { Mean (SD) }\end{array}$}} & \multirow{3}{*}{$\begin{array}{c}p \\
\text { SBP }\end{array}$} & \multirow{3}{*}{$\begin{array}{c}p \\
\text { DBP }\end{array}$} \\
\hline & & & & & & & & \\
\hline & & SBP (mm Hg) & DBP (mm Hg) & & SBP (mm Hg) & DBP (mm Hg) & & \\
\hline 6 & 61 & $98(8)$ & $70(9)$ & 63 & 99 (9) & $70(9)$ & 0.632 & 0.451 \\
\hline 7 & 79 & $101(8)$ & $72(8)$ & 75 & $102(10)$ & $69(8)$ & 0.540 & 0.069 \\
\hline 8 & 75 & $105(9)$ & $70(10)$ & 79 & $103(9)$ & $73(9)$ & 0.125 & 0.018 \\
\hline 9 & 81 & $105(9)$ & $71(7)$ & 79 & $107(9)$ & $75(8)$ & 0.122 & 0.009 \\
\hline 10 & 71 & $107(11)$ & $73(9)$ & 77 & $107(10)$ & $73(9)$ & 0.986 & 0.906 \\
\hline 11 & 76 & $106(11)$ & $70(10)$ & 83 & $105(12)$ & $69(9)$ & 0.723 & 0.607 \\
\hline 12 & 76 & $104(13)$ & $67(10)$ & 72 & $103(10)$ & $66(11)$ & 0.794 & 0.313 \\
\hline 13 & 76 & $101(10)$ & $64(9)$ & 78 & $102(9)$ & $63(10)$ & 0.712 & 0.258 \\
\hline 14 & 72 & $108(12)$ & $67(10)$ & 84 & $106(9)$ & $68(9)$ & 0.230 & 0.789 \\
\hline 15 & 92 & $114(12)$ & $70(12)$ & 99 & $105(9)$ & $67(10)$ & 0.000 & 0.015 \\
\hline 16 & 84 & $112(11)$ & $69(9)$ & 77 & $108(9)$ & $68(8)$ & 0.015 & 0.701 \\
\hline 17 & 56 & $113(11)$ & $69(8)$ & 60 & $109(10)$ & $67(9)$ & 0.093 & 0.170 \\
\hline 18 & 51 & $119(12)$ & $76(11)$ & 46 & $111(11)$ & $70(7)$ & 0.002 & 0.002 \\
\hline
\end{tabular}

SBP-systolic blood pressure, DBP - diastolic blood pressure, SD - standard deviation, $n$ - number

When the mean difference between each age of the same sex was analysed, in boys, an increase in SBP (2.25 mm Hg/year) was observed until the age of 10 years. However, this increase was statistically significant $(p<0.05)$ from 6 to 7 and 7 to 8 year age groups. From 10 years of age SBP reduced $(2 \mathrm{~mm} \mathrm{Hg} /$ year) until the age of 13 years, but this reduction from one age group to the next was not statistically significant. From 13 years onwards SBP increased $6.5 \mathrm{~mm} \mathrm{Hg} /$ year up to 15 year age group. After this statistically significant $(p<0.05)$ increase $(6 \mathrm{~mm} \mathrm{Hg})$ was observed between 17 and 18 year age groups. DBP showed 
a statistically significant $(p<0.05)$ increase from 13-14 years and from $17-18$ years.

In girls the mean SBP increased $(2 \mathrm{~mm} \mathrm{Hg} /$ year $)$ until 10 years and then it decreased $(1.66 \mathrm{~mm}$ $\mathrm{Hg} /$ year) until 13 years of age and increased by $1.8 \mathrm{~mm} \mathrm{Hg} /$ year) thereafter. But this increase from one age to the next is statistically significant $(p<0.05)$ in 8 to 9 year age groups and 13 to 14 year age groups only. The DBP showed a statistically significant $(p<0.05)$ increase from one age to the next in 7 to 8 years and 13 to 14 years. However, from 6 to 10 years of age DBP increases and then there is a reduction until 13 years of age and DBP increases again until 18 years of age. Similar variation in DBP was observed in both boys and girls.

From the age of 15 years, boys had observably higher SBP than girls of the same age which was statistically significant $(p<0.05)$. Considering the DBP, girls in the ages of 8 to 9 years had significantly higher values than boys of the same age.

When the difference between mean DBP of each age group of boys was analysed, statistically significant $(p<0.05)$ increase in DBP was observed from 13 to 14 year and from 17 to 18 years. In girls, statistically significant $(p<0.05)$ increase from one age to the next is observed in 7 to 8 years and 13 to 14 years of age groups. However, from 6 to 10 years of age DBP increases and then there is a reduction until 13 years of age and increases again until 18 years of age. This pattern was observed in both boys and girls.

Pearson correlation between anthropometric measurements and BP are summarised in Table 3.

Table 3: Correlation coefficient (r) of BP with anthropometric measurements

\begin{tabular}{|l|c|c|c|c|}
\hline \multirow{2}{*}{ Anthropometric measurements } & \multicolumn{2}{|c|}{ BOYS } & \multicolumn{2}{c|}{ GIRLS } \\
\cline { 2 - 5 } & SBP & DBP & SBP & DBP \\
\hline Age & $0.382^{*}$ & -0.011 & $0.208^{*}$ & $-0.167^{*}$ \\
\hline Height & $0.461^{*}$ & 0.042 & $0.277^{*}$ & $-0.107^{*}$ \\
\hline Weight & $0.522^{*}$ & $0.134^{*}$ & $0.351^{*}$ & -0.034 \\
\hline BMI & $0.462^{*}$ & $0.174^{*}$ & $0.356^{*}$ & 0.037 \\
\hline Waist circumference & $0.460^{*}$ & $0.114^{*}$ & $0.334^{*}$ & 0.036 \\
\hline Hip circumference & $0.513^{*}$ & $0.125^{*}$ & $0.334^{*}$ & -0.018 \\
\hline
\end{tabular}

* indicates $p<0.001$

Statistically significant $\quad(p<0.001)$ positive correlations of SBP were observed with age, height, weight, BMI, WC and $\mathrm{HC}$ of both boys and girls. In boys, DBP had a significant small positive correlation with weight, BMI, WC and HC, whereas in girls DBP had a statistically significant small negative correlation with age and height.

Stepwise regression analysis was done to derive prediction equations for SBP and DBP based on age, height $(\mathrm{H})$, weight $(\mathrm{W})$, and $\mathrm{BMI}$.

SBP (male) $=0.201 \mathrm{H}+0.408 \mathrm{~W}-1.06$ age +75.54 $\mathrm{R}^{2}=0.281, \quad \mathrm{SEE}= \pm 10.11$

$\mathrm{SBP}($ female $)=0.943 \mathrm{BMI}+0.209 \mathrm{H}-0.75 \mathrm{age}+$ $68.454 \quad \mathrm{R}^{2}=0.143, \quad \mathrm{SEE}= \pm 9.37$

$\mathrm{DBP}($ male $)=0.28 \mathrm{~W}-1.08$ age $+71.637 \quad \mathrm{R}^{2}=$ $0.058, \quad \mathrm{SEE}= \pm 9.45$

$\mathrm{DBP}($ female $)=0.641$ BMI- 0.852 age +68.6 $\mathrm{R}^{2}=0.06, \quad \mathrm{SEE}= \pm 9.25$

As can be seen, the regression analysis of the entire data seems to be unreliable because of the very low value of $R^{2}$. This may be due to the variation of blood pressure of different age groups.

A normogram was constructed with age and height to keep in line with the current practice of diagnosing hypertension in children and adolescents using the data of each age group separately. However, the present study showed better correlation between BP and body weight than with height.

Height percentile values $\left(25^{\text {th }}, 50^{\text {th }}, 75^{\text {th }}\right)$ of each age group are summarised in Table 4.

The $50^{\text {th }}, 90^{\text {th }}, 95^{\text {th }}$ values of both SBP and DBP were analysed according to height percentiles $\left(25^{\text {th }}\right.$, $50^{\text {th }}, 75^{\text {th }}$ ) of each age group and summarised in Table 5. Both $90^{\text {th }}$ and $95^{\text {th }}$ percentiles of BP were derived as the definitions for hypertension and prehypertension are based on those percentiles respectively.

Tanner scale staging was analysed and summarised in Table 6. 
Table 4: Percentile values of height according to age

\begin{tabular}{|c|c|c|c|c|c|c|c|c|}
\hline \multirow[b]{3}{*}{ Age } & \multirow[b]{3}{*}{ No } & \multicolumn{3}{|c|}{ BOYS } & \multirow[b]{3}{*}{ No } & \multicolumn{3}{|c|}{ GIRLS } \\
\hline & & \multicolumn{3}{|c|}{ Height $(\mathrm{cm})$ percentile } & & \multicolumn{3}{|c|}{ Height $(\mathrm{cm})$ percentile } \\
\hline & & 25th & $\mathbf{5 0}^{\text {th }}$ & 75th & & 25th & $50^{\text {th }}$ & 75th \\
\hline 6 & 61 & 115.8 & 119 & 123 & 63 & 116.5 & 120 & 124 \\
\hline 7 & 79 & 119.5 & 124 & 128 & 75 & 121 & 124 & 127.5 \\
\hline 8 & 75 & 127 & 130 & 131.5 & 79 & 125 & 128 & 130.5 \\
\hline 9 & 81 & 130 & 131.5 & 135.5 & 79 & 130 & 131.5 & 135 \\
\hline 10 & 71 & 131.5 & 134 & 138 & 77 & 131.5 & 136 & 140.3 \\
\hline 11 & 76 & 136.2 & 140.3 & 146.5 & 83 & 136 & 142 & 146 \\
\hline 12 & 76 & 141.6 & 145.3 & 149.4 & 72 & 143 & 148.5 & 153.4 \\
\hline 13 & 76 & 144.6 & 150.3 & 157.75 & 78 & 146.9 & 152 & 156.1 \\
\hline 14 & 72 & 151 & 159.3 & 164 & 84 & 151.5 & 154.8 & 159 \\
\hline 15 & 92 & 159 & 163.3 & 167.9 & 99 & 151.5 & 155 & 160.5 \\
\hline 16 & 84 & 162 & 166.9 & 171 & 77 & 153.8 & 158.5 & 162 \\
\hline 17 & 56 & 165.6 & 168.8 & 173.8 & 60 & 153 & 157.5 & 161.5 \\
\hline 18 & 51 & 167.5 & 172 & 175 & 46 & 154.9 & 158.3 & 162.6 \\
\hline
\end{tabular}

Table 5: Reference values of blood pressure according to age and height

\begin{tabular}{|c|c|c|c|c|c|c|c|c|c|c|c|c|c|}
\hline \multirow{3}{*}{$\begin{array}{c}\text { Age } \\
\text { (years) }\end{array}$} & \multirow{3}{*}{$\begin{array}{c}\text { BP } \\
\text { centile }\end{array}$} & \multicolumn{6}{|c|}{ Boys } & \multicolumn{6}{|c|}{ Girls } \\
\hline & & \multicolumn{3}{|c|}{$\begin{array}{c}\text { Systolic BP } \\
\text { Height percentile }\end{array}$} & \multicolumn{3}{|c|}{$\begin{array}{c}\text { Diastolic BP } \\
\text { Height percentile }\end{array}$} & \multicolumn{3}{|c|}{$\begin{array}{c}\text { Systolic BP } \\
\text { Height percentile }\end{array}$} & \multicolumn{3}{|c|}{$\begin{array}{c}\text { Diastolic BP } \\
\text { Height percentile }\end{array}$} \\
\hline & & 25th & 50th & 75th & 25th & 50th & 75th & 25th & 50th & 75th & 25th & 50th & 75th \\
\hline \multirow[t]{3}{*}{6} & $50^{\text {th }}$ & 95 & 99 & 101 & 69 & 71 & 77 & 95 & 99 & 102 & 70 & 68 & 73 \\
\hline & $90^{\text {th }}$ & 110 & 110 & 112 & 80 & 82 & 82 & 112 & 112 & 117 & 80 & 81 & 83 \\
\hline & $95^{\text {th }}$ & 114 & 114 & 116 & 82 & 84 & 85 & 118 & 117 & 119 & 82 & 83 & 85 \\
\hline \multirow[t]{3}{*}{7} & $50^{\text {th }}$ & 98 & 101 & 102 & 70 & 73 & 72 & 99 & 102 & 104 & 66 & 70 & 74 \\
\hline & $90^{\text {th }}$ & 109 & 109 & 115 & 80 & 82 & 81 & 106 & 116 & 122 & 74 & 81 & 82 \\
\hline & $95^{\text {th }}$ & 113 & 113 & 118 & 84 & 84 & 85 & 111 & 118 & 132 & 77 & 92 & 86 \\
\hline \multirow[t]{3}{*}{8} & $50^{\text {th }}$ & 103 & 104 & 107 & 70 & 66 & 70 & 100 & 101 & 108 & 74 & 73 & 75 \\
\hline & $90^{\text {th }}$ & 114 & 115 & 127 & 79 & 85 & 84 & 109 & 116 & 118 & 85 & 86 & 86 \\
\hline & $95^{\text {th }}$ & 116 & 124 & 128 & 87 & 88 & 92 & 112 & 120 & 131 & 86 & 92 & 86 \\
\hline \multirow[t]{3}{*}{9} & $50^{\text {th }}$ & 101 & 105 & 108 & 72 & 72 & 71 & 102 & 110 & 113 & 72 & 73 & 82 \\
\hline & $90^{\text {th }}$ & 114 & 115 & 125 & 81 & 81 & 81 & 113 & 122 & 128 & 81 & 85 & 88 \\
\hline & $95^{\text {th }}$ & 119 & 123 & 126 & 83 & 82 & 83 & 115 & 124 & 130 & 83 & 87 & 90 \\
\hline \multirow[t]{3}{*}{10} & $50^{\text {th }}$ & 103 & 106 & 112 & 76 & 72 & 73 & 107 & 104 & 111 & 70 & 71 & 76 \\
\hline & $90^{\text {th }}$ & 119 & 122 & 127 & 83 & 84 & 89 & 121 & 120 & 126 & 82 & 83 & 90 \\
\hline & $95^{\text {th }}$ & 122 & 123 & 132 & 89 & 86 & 93 & 122 & 130 & 134 & 86 & 93 & 96 \\
\hline \multirow[t]{3}{*}{11} & $50^{\text {th }}$ & 104 & 103 & 112 & 66 & 73 & 74 & 96 & 106 & 107 & 68 & 70 & 67 \\
\hline & $90^{\text {th }}$ & 116 & 118 & 125 & 78 & 80 & 82 & 120 & 122 & 128 & 80 & 82 & 84 \\
\hline & $95^{\text {th }}$ & 121 & 125 & 126 & 80 & 83 & 85 & 129 & 126 & 134 & 82 & 84 & 90 \\
\hline \multirow[t]{3}{*}{12} & $50^{\text {th }}$ & 100 & 102 & 111 & 67 & 66 & 68 & 99 & 99 & 113 & 65 & 66 & 71 \\
\hline & $90^{\text {th }}$ & 119 & 117 & 129 & 75 & 80 & 88 & 104 & 116 & 118 & 73 & 81 & 81 \\
\hline & $95^{\text {th }}$ & 120 & 130 & 140 & 78 & 89 & 90 & 110 & 118 & 125 & 73 & 81 & 90 \\
\hline \multirow[t]{3}{*}{13} & $50^{\text {th }}$ & 95 & 101 & 109 & 62 & 65 & 65 & 100 & 102 & 102 & 62 & 62 & 66 \\
\hline & $90^{\text {th }}$ & 110 & 115 & 116 & 76 & 76 & 76 & 108 & 117 & 118 & 69 & 79 & 79 \\
\hline & $95^{\text {th }}$ & 111 & 120 & 121 & 77 & 80 & 80 & 113 & 130 & 123 & 77 & 81 & 86 \\
\hline \multirow[t]{3}{*}{14} & $50^{\text {th }}$ & 97 & 109 & 115 & 65 & 68 & 69 & 105 & 105 & 111 & 70 & 70 & 74 \\
\hline & $90^{\text {th }}$ & 115 & 126 & 132 & 79 & 77 & 90 & 115 & 119 & 119 & 79 & 75 & 82 \\
\hline & $95^{\text {th }}$ & 122 & 131 & 135 & 80 & 79 & 90 & 117 & 124 & 126 & 80 & 80 & 83 \\
\hline \multirow[t]{3}{*}{15} & $50^{\text {th }}$ & 105 & 114 & 120 & 68 & 70 & 73 & 102 & 105 & 107 & 68 & 67 & 67 \\
\hline & $90^{\text {th }}$ & 128 & 130 & 136 & 82 & 86 & 94 & 116 & 119 & 122 & 78 & 80 & 81 \\
\hline & $95^{\text {th }}$ & 130 & 130 & 145 & 85 & 90 & 104 & 122 & 127 & 127 & 84 & 83 & 83 \\
\hline \multirow[t]{3}{*}{16} & $50^{\text {th }}$ & 107 & 112 & 121 & 63 & 71 & 70 & 103 & 110 & 107 & 65 & 70 & 68 \\
\hline & $90^{\text {th }}$ & 121 & 122 & 142 & 76 & 82 & 84 & 116 & 119 & 124 & 72 & 80 & 84 \\
\hline & $95^{\text {th }}$ & 124 & 128 & 144 & 82 & 84 & 90 & 119 & 127 & 133 & 75 & 82 & 89 \\
\hline \multirow[t]{3}{*}{17} & $50^{\text {th }}$ & 109 & 110 & 119 & 69 & 68 & 72 & 104 & 109 & 108 & 67 & 66 & 70 \\
\hline & $90^{\text {th }}$ & 126 & 132 & 134 & 81 & 79 & 87 & 121 & 124 & 126 & 83 & 78 & 82 \\
\hline & $95^{\text {th }}$ & 134 & 138 & 139 & 82 & 83 & 88 & 127 & 126 & 127 & 87 & 84 & 85 \\
\hline \multirow[t]{3}{*}{18} & $50^{\text {th }}$ & 116 & 115 & 123 & 75 & 75 & 76 & 105 & 112 & 115 & 69 & 71 & 71 \\
\hline & $90^{\text {th }}$ & 134 & 140 & 140 & 89 & 91 & 98 & 122 & 128 & 135 & 78 & 79 & 80 \\
\hline & $95^{\text {th }}$ & 135 & 142 & 141 & 94 & 104 & 100 & 123 & 130 & 136 & 80 & 81 & 83 \\
\hline
\end{tabular}


Table 6: Mean age, height and blood pressure according to Tanner scale

\begin{tabular}{|c|c|c|c|c|c|c|c|c|}
\hline \multirow{3}{*}{ Stages } & \multicolumn{8}{|c|}{ Boys } \\
\hline & \multicolumn{4}{|c|}{ Pubic hair } & \multicolumn{4}{|c|}{ Penile development } \\
\hline & No. & Age (SD) & SBP & DBP & No. & Age (SD) & SBP & DBP \\
\hline Stage 1 & 275 & $10.8(1.5)$ & $106(10)$ & $69(10)$ & 173 & $10.7(2.0)$ & $106(11)$ & $71(9)$ \\
\hline Stage 2 & 90 & $12.6(1.8)$ & $103(12)$ & $68(10)$ & 166 & $11.7(1.7)$ & $107(12)$ & $69(10)$ \\
\hline Stage 3 & 106 & $13.5(1.8)$ & $106(13)$ & $67(9)$ & 147 & $13.6(2.0)$ & $105(13)$ & $66(11)$ \\
\hline Stage 4 & 216 & $16.0(1.3)$ & $114(12)$ & $71(10)$ & 177 & $15.7(1.6)$ & $113(12)$ & $71(10)$ \\
\hline Stage 5 & 53 & $16.5(1.3)$ & $115(11)$ & $69(10)$ & 77 & $16.4(1.1)$ & $113(10)$ & $69(9)$ \\
\hline \multirow{3}{*}{ Stages } & \multicolumn{8}{|c|}{ Girls } \\
\hline & \multicolumn{4}{|c|}{ Pubic hair } & \multicolumn{4}{|c|}{ Breast development } \\
\hline & No. & Age (SD) & SBP & DBP & No. & Age (SD) & SBP & DBP \\
\hline Stage 1 & 136 & $9.8(1.1)$ & $106(10)$ & $73(9)$ & 99 & $9.8(1.1)$ & $104(11)$ & $71(8)$ \\
\hline Stage 2 & 135 & $11.1(1.2)$ & $104(11)$ & $69(10)$ & 115 & $10.5(1.2)$ & $106(11)$ & $74(10)$ \\
\hline Stage 3 & 97 & $12.9(1.5)$ & $104(10)$ & $64(11)$ & 118 & $12.2(1.6)$ & $105(10)$ & $65(10)$ \\
\hline Stage 4 & 256 & $15.0(1.6)$ & $107(10)$ & $68(10)$ & 320 & $14.9(1.8)$ & $106(9)$ & $67(10)$ \\
\hline Stage 5 & 131 & $15.9(1.6)$ & $108(10)$ & $68(8)$ & 103 & $15.9(1.5)$ & $111(10)$ & $68(8)$ \\
\hline
\end{tabular}

Based on the pubic hair stage, the SBP of boys decreases from stage 1 to stage 2 and thereafter increases from stage 3 until stage 5 . Tanner staging based on penile development shows that there is 1 $\mathrm{mm} \mathrm{Hg}$ increase in SBP from stage 1 to stage 2, 2 $\mathrm{mm} \mathrm{Hg}$ reduction from stage 2 to stage 3 and then there is $8 \mathrm{~mm} \mathrm{Hg}$ increase from stage 3 to stage 4 . However, statistical significance $(p<0.05)$ was observed only between stage 3 and stage 4 . In girls, there is a reduction of BP from stage 1 to stage 2 according to pubic hair stage, but, in contrast, breast developmental stage shows an increase in BP from stage 1 to stage 2. Statistical significance $(p<0.05)$ in SBP was observed only from stage 3 to stage 4 based on breast development stage. In both boys and girls, there was a reduction in DBP from stage 1 to stage 3 and then it increased in stage 4. However, these differences were not statistically significant.

Statistically significant correlations were observed between SBP and Tanner staging by pubic hair $(0.337)$ and penile developments $(0.287)$ in boys. In girls SBP had correlations $(p<0.05)$ of 0.173 , 0.214 with pubic hair and breast development. Respective correlations $(p<0.05)$ with DBP were $0.168,-0.186$.

\section{Discussion}

In this study population, mean BP increases from 6 to 10 years of age and then there is a reduction until 13 years followed by an increase up to the age of 18 years. A similar pattern can be noticed in the SBP and DBP of both boys and girls. A study done in Madhya Pradesh, India showed a continuous increase in SBP from 7 to 14 years of age ${ }^{10}$. Children from Calcutta, India showed a different pattern $^{11}$. In boys, there was a decrease in SBP from 6 to 9 years and then a gradual increase whilst in girls SBP gradually increased with drops in 7 and 10 years ${ }^{11}$. This variation in SBP among children from Calcutta and Jaffna suggests that there may be some factor influencing SBP during these ages. This could be due to pubertal or environmental factors. There is no documented reason for this fluctuation in BP. There is a coincidental factor affecting children of 9-10 years in Sri Lanka because they have to sit for a highly competitive scholarship examination which determines grade 6 admission in many schools. Intense coaching for this examination starts even when they are in grade 5. This factor was not anticipated at the time of planning the study. Another study may be needed to see whether the stress of this examination affects the BP.

When the mean SBP was compared with that of Indian children from two studies ${ }^{10,11}$, it is seen that up to the age of 11 years, SBP was higher in the present population than in Indian children. However, from 12 years of age, the SBP of Sri Lankan Tamil children was lower than that of the Indian children. This was observed in both boys and girls. DBP was higher in Sri Lankan boys than in Indian boys. However, after 10 years of age, DBP was lower in the present study than in the children from Calcutta. In girls, DBP was lower in the present study after 12 years of age than in the children of both Indian studies. These observations appear to confirm the ethnic differences in BP. 
Mean SBP and DBP of Sinhalese boys of the 6-10 year age group at a purana village were $93.5 \pm 2.1$ $\mathrm{mmHg}$ and $62.3 \pm 1.5 \mathrm{mmHg}^{6}$. When we calculated the respective means, they were $103.5 \pm 9.4$ and $71.1 \pm 8.8 \mathrm{mmHg}$ respectively. Similarly, mean SBP and DBP of Sinhalese boys of 11-15 year age group at a purana village were $96.7 \pm 1.8 \mathrm{mmHg}$ and $63.8 \pm 2.2 \mathrm{mmHg}^{6}$. In the current study the mean values of boys were $107.0 \pm 12.6 \mathrm{mmHg}$ and $67.8 \pm 10.2 \mathrm{mmHg}$ respectively. Girls of the $6-10$ year age group at a purana village had mean SBP and DBP of $97.7 \pm 2.3 \mathrm{mmHg}$ and $64.7 \pm 1.9 \mathrm{mmHg}$ respectively ${ }^{6}$. In the current study the values were $103.8 \pm 9.7 \mathrm{mmHg}$ and $72.1 \pm 8.8 \mathrm{mmHg}$. Similarly, mean SBP and DBP of Sinhalese girls of 11-15 year age group were $110.4 \pm 3.8 \mathrm{mmHg}$ and $72.1 \pm 2.9 \mathrm{mmHg}$. The present values were $104.4 \pm 10$ $\mathrm{mmHg}$ and $66.6 \pm 10 \mathrm{mmHg}$ respectively. This shows that Jaffna boys up to 15 years and girls up to 10 years have higher BP than Sinhalese children at a purana village. After these ages, both SBP and DBP of the current study population were lower than Sinhalese children. In the study at a purana village, BP was recorded only once and DBP was recorded at muffling of sounds. These two factors may explain the higher values than the present study although the life style and food habits of the purana villagers would also have affected the BP.

Statistically significant increase in SBP and DBP observed in boys in the ages of 13 to 15 years coincides with the higher rate of somatic growth $(6 \mathrm{~cm} /$ year) during this age. But in girls statistically significant increase was observed only in 13-14 years. This observation agrees with the findings of Wanzu et $a l^{12}$ who reported that the BP increases in puberty more in males than females. Observably higher BP in boys than the girls of same age after 15 years old suggests that boys attain puberty during this stage which influences the BP of boys. Higher DBP in girls than the boys in the ages of 7 to 9 years also may be due to influence of prepubertal factors. Increase in BP from stage 3 of Tanner staging scale to stage 4 shows that the puberty influences the BP.

This study was done in a large number of healthy children and adolescents. There is no previously published data of BP values available for healthy Tamil children in Sri Lanka. The Normogram developed can be used in clinical practice.

\section{Conclusions}

BP of healthy Sri Lankan Tamil children in the Jaffna district has significant correlations with anthropometric measurements and Tanner staging.

\section{Acknowledgements}

The authors acknowledge technical officers, Mr. S. B. Rameshkumar and Mr. Pavalarajah and other staff at the Department of Physiology for their assistance.

\section{References}

1. Pickering TG, Hall JE, Appel LJ, Falkner BE, Graves J, Hill MN, et al. Recommendations for blood pressure measurement in humans and experimental animals. Circulation 2005; 111(5):697-716. https://doi.org/10.1161/01.CIR.0000154900.76 284.F6

PMid: 15699287

2. Bonita F. Fourth report on the diagnosis, evaluation and treatment of high blood pressure in children and adolescents. NIH Publication No. (96-3790) Revised May 2005. Available at: www.academia.edu/.../Diagnosis_Evaluation and_Treatment_of_High_Blood_Pressure. Accessed on 18th January 2017

3. Elaine M, Lawrence M, Connie EM, Philip RK, Stephen RD, Thomas RK. Relationship between elevated arterial stiffness and increased LV mass in adolescents and young adults. Journal of Pediatrics 2011; 158(5):715-21. https://doi.org/10.1016/j.jpeds.2010.12.020 PMid: 21300369 PMCid: PMC3075322

4. Pande V, Mane S, Agarkhedar S, Prakash G, Singhania S. Blood pressure and blood sugar levels in over weight adolescents from an industrial town of western India. International Journal of Pharmacology and Biomedical Science 2012; 3 (4):229-32.

5. Borade A, Kadam GS, Bhide G, Dhongade R. Study of blood pressure and blood sugar levels in adolescence and comparison with BMI. Indian Journal of Medical Sciences 2011; 65 (7):297-310.

https://doi.org/10.4103/0019-5359.107391

PMid: 23422703

6. Wikramanayake ER, Chandrasekera MS. Variation with sex and age of blood pressure in the inhabitants of a "purana" village in Sri Lanka. Ceylon Journal of Medical Science 1991; 34(1):7-13.

7. Lane DA, Lip DYH. Ethnic differences in hypertension and blood pressure control in the UK. Quarterly Journal of Medicine 2001: 94(7); 391-6. https://doi.org/10.1093/qjmed/94.7.391 
8. Agyemang C, Bhopal RS. Is the blood pressure of South Asian adults in the UK higher or lower than that in European white adults? A review of cross-sectional data. Journal of Human Hypertension 2002; 16(11): 739-51.

https://doi.org/10.1038/sj.jhh.1001488

PMid: 12444535

9. Waist circumference and waist-hip ratio: Report of a WHO Expert Consultation Geneva, 8-11 December 2008.Available at apps.who.int/iris/bit stream/10665/44583/1/9789241501491_eng.p df. Accessed on 12th November 2016.

10. Nazeem I S, Bose S. "Age and height specific normative reference limits of blood pressure in school going children in Indian population". Indian Journal of Basic \& Applied Medical Research.2012; 1(4): 268-78.
11. Bose AS, Marimuthu P, Chakraborty AK. Mean blood pressure values of 3-17 year old children in an urban area. Health and Population-Perspectives and Issues 2000; 23(1):28-36.

12. Wanzhu Tu, Eckert GJ, Saha C, Pratt JH. Synchronization of adolescent blood pressure and pubertal somatic growth. Journal of Clinical Endocrinology and Metabolism 2009; 94(12): 5019-22.

https://doi.org/10.1210/jc.2009-0997

PMid: 19850686 PMCid : PMC2795652 